\title{
Induction of chimerism and immune tolerance using belatacept
}

A new study has demonstrated successful induction of transient mixed chimerism and long-term renal allograft tolerance in cynomolgus monkeys using a conditioning regimen that includes belatacept. This CTLA4-Ig fusion protein blocks T-cell costimulation by binding to B7 molecules expressed on antigen presenting cells.

Researcher Tatsuo Kawai and colleagues have been working on tolerance induction in kidney transplantation for decades.

"In primates, including humans, induction of transient chimerism is sufficient to induce stable renal allograft tolerance," says Kawai. "Our current hypothesis of this tolerance mechanism is that donorspecific T regulatory cells are induced after transient donor chimerism, which is maintained by the kidney allograft."

Kawai and colleagues previously showed that addition of costimulatory blockade using anti-CD154 monoclonal antibody $(\mathrm{mAb})$ to their nonmyeloblative conditioning regimen significantly improved the induction of chimerism and allograft tolerance in nonhuman primates. "As anti-CD154 $\mathrm{mAb}$ is not clinically available, we decided to test the CTLA4-Ig fusion proteins belatacept and abatacept in place of CD154 blockade," explains Kawai. These agents have been approved by the FDA for use in kidney transplantation and rheumatoid arthritis, respectively.

\section{We have successfully developed a clinically applicable regimen for induction of renal allograft tolerance... 77}

The researchers report that a conditioning regimen involving low-dose total body irradiation, thymic irradiation, anti-thymocyte globulin, a 1-month posttransplantation course of ciclosporin and four doses of belatacept $(20 \mathrm{mg} / \mathrm{kg}$ on days 0 and 2 and $10 \mathrm{mg} / \mathrm{kg}$ on days 5 and 15 after kidney transplantation) resulted in the development of lymphoid and myeloid chimerism in four of five recipients, three of which survived long-term ( $>370$ days) without immunosuppression. The level of chimerism achieved with belatacept was significantly higher than that observed with anti-CD154 mAb.

When abatacept was substituted for belatacept in the conditioning regimen, all three of the recipients achieved limited lymphoid chimerism and rejected their allografts after discontinuation of immunosuppression. The researchers suggest that this difference in efficacy might be related to the higher affinity of belatacept than abatacept for the B7 molecule.

"We have successfully developed a clinically applicable regimen for induction of renal allograft tolerance using available modalities," concludes Kawai. "We now plan to conduct a clinical trial in HLAmismatched kidney transplantation using the belatacept regimen."

Ellen F. Carney

Original article Yamada, Y. et al. Use of CTLA4Ig for induction of mixed chimerism and renal allograft tolerance in nonhuman primates. Am. J. Transplant. 14, 2704-2712 (2014) 